\title{
Improving Anticoagulation Therapy Using Point-of-Care Testing and a Standardized Protocol
}

\author{
Curtis A. Franke, $M D^{1}$ \\ Lori M. Dickerson, PharmD ${ }^{2}$ \\ Peter J. Carek, $M D, M S^{2}$ \\ 'Baylor Family Medicine at Uptown, Health \\ Texas Provider Network, Dallas, Texas \\ ${ }^{2}$ Trident/MUSC Family Medicine Residency \\ Program, Department of Family Medicine, \\ Medical University of South Carolina, \\ Charleston, South Carolina
}

\begin{abstract}
PURPOSE Many patients in primary care require anticoagulation with warfarin for the prevention of venous and systemic embolism. Achieving the goal international normalized ratio (INR) with warfarin is challenging. The purpose of this quality improvement initiative was to increase the proportion of patients taking warfarin with an INR value within the goal range.
\end{abstract}

METHODS We included all patients identified on an anticoagulation log in the family medicine residency practice during 3 time periods: baseline, after point-ofcare (POC) testing was initiated (intervention period 1), and after a standardized warfarin-dosing protocol was implemented (intervention period 2). Educational sessions were conducted during each intervention period. Measures included the frequency of INR monitoring and the percentage of office visits in which patients' values were within the goal INR range. Data were analyzed using descriptive statistics, the Student $t$ test, and the $\chi^{2}$ test.

RESULTS At baseline, patients had an average of 2.6 INR tests performed, and $30.8 \%$ were within the INR goal range. Using POC testing, the frequency of monitoring increased to 4.3 INR tests per patient $(P=.04)$, but the percentage of patients within the INR goal remained low at $32.1 \%(P=.88)$. When physicians implemented the standardized protocol to guide warfarin dosing, the frequency of testing was similar (3.8 tests per patient), but the percentage of patients within the INR goal increased to $45.9 \%(P<.04)$.

CONCLUSIONS POC testing increased the frequency of INR testing, and additional use of a standardized protocol for warfarin dosing increased the percentage of patients within the INR goal range. This model of anticoagulation management could be easily implemented in any family medicine office.

Ann Fam Med 2008;6(suppl 1):s28-s32. DOI: 10.1370/afm.739.

\section{INTRODUCTION}

M illions of patients annually receive anticoagulation with a vitamin $\mathrm{K}$ antagonist, specifically warfarin, for the prevention of venous thromboembolism and systemic embolism associated with atrial fibrillation and prosthetic heart valves. First episodes of symptomatic venous thromboembolism occur in approximately 100 persons per 100,000 each year in the United States, with a greater incidence among whites and African Americans than Hispanics and Asian-Pacific Islanders. ${ }^{1}$ Atrial fibrillation, the most common cardiac arrhythmia in the general population, affects nearly 2.5 million people in the United States. ${ }^{2}$

The optimal therapeutic range for warfarin for most indications, including atrial fibrillation, deep venous thrombosis, pulmonary embolism, and prosthetic heart valves, is an international normalized ratio (INR) of 2.0 to 3.0, with a target INR of $2.5 .^{3}$ The recommendations differ for mechanical heart valves, with the target INR being 3.0 (range, 2.5-3.5). ${ }^{4}$ 
Although highly effective, warfarin is a challenge to use in clinical practice because of its narrow therapeutic window, variability in dose response among patients, numerous drug and food interactions, miscommunication about dosing between the patient and physician, and patient nonadherence. ${ }^{3}$ The American College of Chest Physicians (ACCP) provides guidelines for the management of warfarin, including initiation, frequency of monitoring, and management of nontherapeutic INR values. ${ }^{3}$ Even with these guidelines, many patients have subtherapeutic INR values and clinical failures, while others experience supratherapeutic INR values and bleeding episodes. For instance, Samsa et $\mathrm{al}^{5}$ found in their study that only $34 \%$ to $47 \%$ of INR tests conducted by family practices fell strictly within the therapeutic range.

Despite adjusting warfarin dosing to achieve a therapeutic INR, the risk of major bleeding in patients receiving warfarin long term ranges from $1 \%$ to $3 \%$ per year. ${ }^{3,6-8}$ In the Stroke Prevention in Atrial Fibrillation II study, the rate of major bleeding while receiving warfarin was $2.3 \%$ per year and was directly related to the intensity of anticoagulation. ${ }^{9}$ Close monitoring of warfarin therapy with serial INR measurements and clinical assessment of patients are therefore essential to provide safe and effective anticoagulation.

Approaches to improve anticoagulation include the use of point-of-care (POC) testing, warfarin-dosing protocols, anticoagulation management services with dedicated personnel (ie, anticoagulation clinics), and computer software programs to aid in dose adjustments. ${ }^{3,10-19}$ Warfarin-dosing protocols incorporating pharmacogenetic factors (CYP2C9 and VKORC1 genotypes) have been proposed, but testing is not widely available in primary care. ${ }^{20}$ Unfortunately, there are no randomized trials comparing the methods of anticoagulation management, so the best approach to this problem is unknown. Two before-and-after studies have documented that the coordinated approach of an anticoagulation clinic reduces the frequency of major hemorrhage and recurrent thromboembolism when compared with usual care. ${ }^{14,15}$ A comparison of an anticoagulation clinic with usual care by family physicians found a higher frequency of INR values within therapeutic range for patients in the anticoagulation clinic. ${ }^{17}$ Many primary care clinicians and patients will not have access to an existing anticoagulation management service, however.

Two methods that are easily adopted in most family practices include the use of POC testing and the use of warfarin-dosing protocols. The accuracy of POC INR testing compared with standard measurement in a hospital laboratory is variable. ${ }^{10,21-24}$ Despite this variability, POC measurement results in the same clinical decision in $66 \%$ to $78 \%$ of cases when compared with decisions based on the results from a hospital-based laboratory. ${ }^{10}$ POC testing has furthermore proven to be an effective monitoring modality with resulting beneficial clinical outcomes. ${ }^{25,26}$ This testing provides clinicians with immediate results, allowing for same-day medication adjustments and direct communication between physician and patient regarding management. Patients report a preference for POC testing, as they have less pain on visual analog scales and spend an average of 33 minutes less time in the clinic. ${ }^{27}$ Finally, POC testing may offer additional revenue sources for physicians in an era of declining reimbursement for physician services. ${ }^{28}$

Warfarin-dosing protocols are recommended tools to provide a systematic approach to managing anticoagulation and adjusting warfarin doses. ${ }^{3}$ Such protocols (paper-based or computerized software models) are used in anticoagulation management services and patient self-monitoring models, but there are no randomized trials comparing different dosage adjustment algorithms. ${ }^{3,16,19,29}$

Given the frequency of use of warfarin and the known difficulties with appropriate anticoagulation, the Trident/Medical University of South Carolina (MUSC) Family Medicine residency practice undertook a quality improvement initiative to increase the proportion of patients taking warfarin with an INR value within the goal range. The initiative included using POC testing and a standardized warfarin-dosing protocol.

The Trident/MUSC Family Medicine Residency Program is a community-based, university-affiliated residency program located in a large community hospital approximately 20 miles from the academic medical center (MUSC). To enhance continuity of care in the residency practice, individual residents are scheduled during the same patient care sessions throughout the year. In addition, 3 attending physicians have an office practice in the residency program. Although an anticoagulation clinic is available for referral at MUSC, the residency program wanted to provide an educational system for management of this patient population within the training setting.

\section{METHODS}

Beginning in June 2004, all patients visiting the residency practice who received INR testing were tracked by the office laboratory using an anticoagulation encounter $\log$, managed by the medical laboratory technician (MLT). In this quality improvement initiative, all patients identified on the anticoagulation encounter log were included to determine (1) the frequency of INR monitoring and (2) the proportion of office visits in which patients were within the goal INR range. There were no exclusion criteria, and 
every visit wherein an INR was drawn was included in the data set. The quality improvement team included the MLT, an upper-level resident (C.A.F.), and the faculty mentors (L.M.D. and P.J.C.).

Baseline data on the above measures were collected from June through September 2004. During this time period, all INR tests were sent out to the hospital laboratory for analyses, and all warfarin dosage adjustments and follow-up visits were arranged by the primary clinician without any formalized process. In October 2004, the practice switched to an in-office INR POC testing device (Protime Microcoagulation System, ITC, Edison, New Jersey). Residents and faculty participated in a didactic educational session about warfarin monitoring with the POC device. Data were collected from October 2004 through January 2005 (intervention period 1). POC testing throughout the quality improvement initiative was performed by the same MLT.

From February 2005 until August 2005, the quality improvement team reviewed the literature and identified the standardized warfarin-dosing protocol (Figure 1). In September 2005, the protocol was implemented in the residency practice. The protocol was developed based on the ACCP guidelines and was presented at a didactic educational session to residents and faculty in that month. It was attached to each INR result generated from the POC testing in the practice and was given to the ordering physician for management. Physicians were asked to mark the current warfarin dosage, current INR value, new warfarin dosage, and scheduled follow-up care on the protocol sheet. The MLT collected each of these INR results and accompanying protocol documents for analyses. The resident author
(C.A.F.) reviewed warfarin dosages and patient followup to determine if each adhered to the protocol. Data were collected from October 2005 through January 2006 (intervention period 2).

All data were entered into a Microsoft Excel spreadsheet (Microsoft Corp, Redmond, Washington) by one author (C.A.F.). Data were analyzed using descriptive statistics, the Student $t$ test, and the $\chi^{2}$ test. This project was approved as exempt research by the Institutional Review Board at MUSC.

\section{RESULTS}

Patient demographics and indications for warfarin therapy did not differ between the 2 intervention periods (Table 1). None of the patients studied had documented hypercoagulable states.

During the 4-month baseline period (June through September 2004), 30 patients had 78 INR tests analyzed at the send-out hospital laboratory (mean, 2.6 tests per patient). The proportion of INR values within the goal range was $30.8 \%$.

During the first 4 -month intervention period (October 2004 through January 2005), which took place after POC testing had been initiated in the office, 37 patients had 159 INR tests recorded in the encounter $\log$. Although the frequency of INR testing increased (2.6 tests per patient during the baseline period vs 4.3 tests per patient during intervention period 1 , $P=.04)$, this increase did not translate into a significant improvement from baseline in the proportion of INR values within the goal range $(30.8 \%$ vs $32.1 \%, P=.88)$.

During the second 4 -month intervention period

\section{Figure 1. Warfarin (Coumadin)-dosing protocol.}

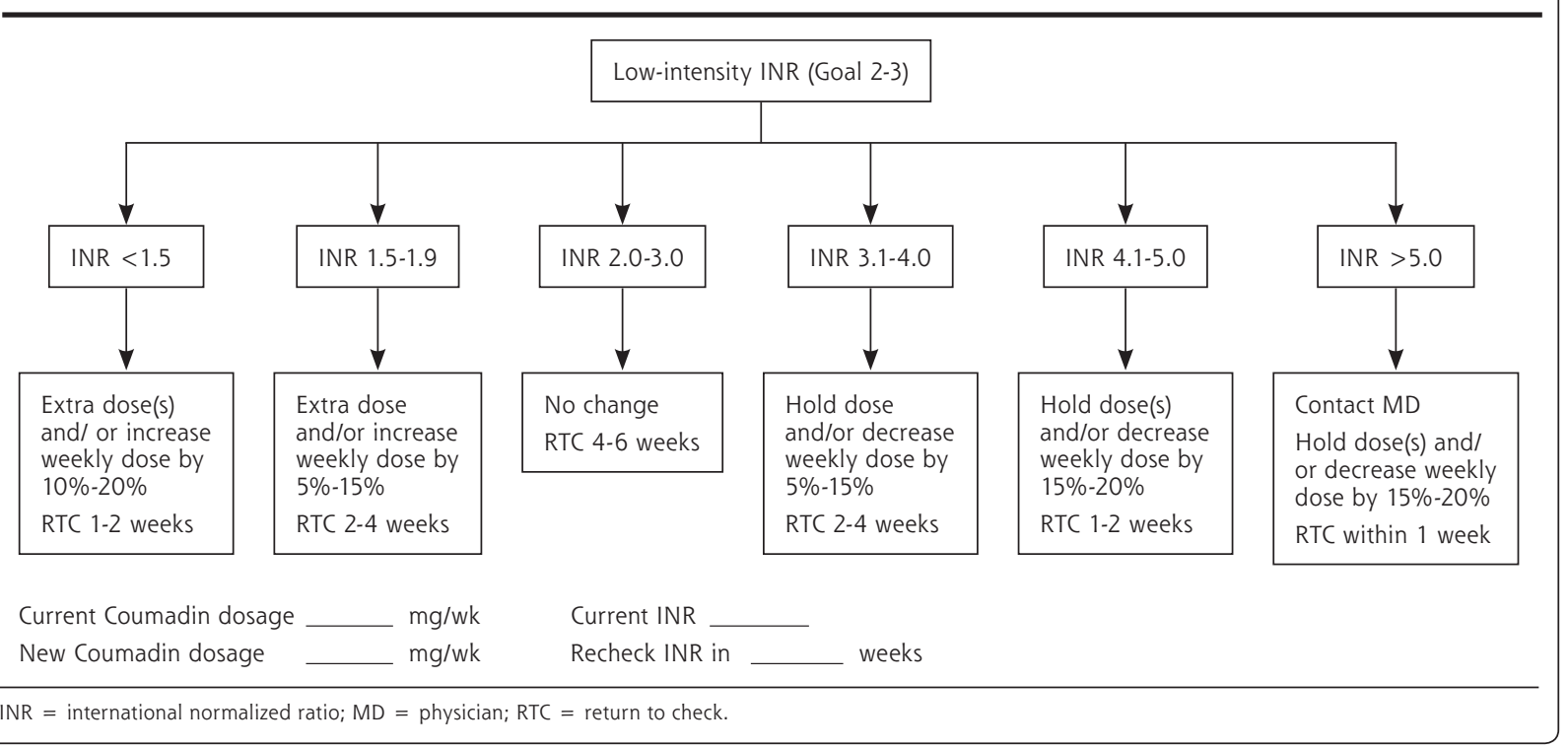




\section{Table 1. Patient Demographic Characteristics}

\begin{tabular}{lccc}
\hline Characteristic & $\begin{array}{c}\text { Intervention } \\
\text { Period } \mathbf{1}(\mathbf{n}=\mathbf{3 7})\end{array}$ & $\begin{array}{c}\text { Intervention } \\
\text { Period 2 }(\mathbf{n}=\mathbf{3 5})\end{array}$ & P Value \\
\hline Age, mean (SD), years & $58.97(14.75)$ & $61.81(14.51)$ & .44 \\
Sex, No. (\%) & $19(55.9)$ & $15(48.4)$ & .55 \\
$\quad$ Female & $15(44.1)$ & $16(51.6)$ & \\
$\quad$ Male & & & \\
Indication for warfarin & $21(61.8)$ & $17(54.8)$ & .95 \\
therapy, No. (\%) & $9(26.5)$ & $10(32.3)$ & \\
DVT and/or PE & $3(8.8)$ & $3(9.7)$ & \\
Atrial fibrillation & $1(2.9)$ & $1(3.2)$ & \\
Prosthetic heart valve & & \\
Other & & \\
\hline DVT = deep vein thrombosis; PE = pulmonary embolism. & \\
\hline
\end{tabular}

45.9\%). The frequency of follow-up, however, was arranged according to the protocol in only $51 \%$ of cases.

Only $73 \%$ of the standardized warfarin-dosing protocol forms were completed in the quality improvement initiative. Physicians may have used the protocol to make appropriate adjustments but simply did not complete the forms. On the other hand, physicians may have had difficulty interpreting the protocol and therefore left the form blank. Follow-up from the MLT during each patient care session might have resulted in more complete data for analysis. Although the protocol suc-

(October 2005 through January 2006), the standardized protocol was attached to 133 INR tests from 35 patients. The MLT collected $100 \%$ of the protocol documents. Physicians completed 97 (73\%) of the protocol sheets, including information about current warfarin dosage, current INR value, new warfarin dosage, and recommendation for follow-up INR testing. Using the standardized protocol, 113 (85\%) of the warfarin dosages were adjusted correctly and physicians arranged appropriate INR follow-up for 68 (51\%) of tests. The use of the standardized protocol did not increase the frequency of INR measurement (4.3 tests per patient during intervention period 1 vs 3.8 tests per patient during intervention period 2, $P=.51$ ), but it did increase the proportion of INR values within the goal range to $45.9 \%(P<.05$ vs intervention period 2$)$.

\section{DISCUSSION}

Our results are similar to those found in other anticoagulation improvement efforts. Gill and Landis ${ }^{18}$ used POC testing and anticoagulation dosage adjustments with a physician-approved algorithm in cardiology patients and found an increase in the percentage of in-range INRs from $40.7 \%$ to $58.5 \%$. Wilson and colleagues ${ }^{17}$ compared anticoagulation care in 221 patients randomized to an anticoagulation clinic or their family physician's practice. They found a modest improvement in the proportion of patients within the therapeutic range who were managed by the anticoagulation clinic ( $82 \%$ vs $76 \%$ ), but also found more INR measurements by the family physicians ( 13 vs 11 over 3 months).

In this study, POC testing alone increased the frequency of INR testing but did not improve the proportion of INR values within the goal range. The addition of standardized warfarin dosing to POC testing resulted in appropriate warfarin-dosing adjustment in $85 \%$ of cases and improved the proportion of INR values within the goal range by $15 \%$ (from $30.8 \%$ to cessfully guided the anticoagulation dosing in $85 \%$ of cases, physicians had more difficulty with arranging follow-up INR monitoring. Frequency of follow-up is clearly noted in the protocol, but physicians may have simply focused on calculating the new warfarin dosage.

This study is limited by several factors. Patients were identified when they visited the office, had an INR value measured, and were entered on the anticoagulation encounter log by the MLT. It is therefore possible that some patients were not included in the quality improvement initiative because they had their anticoagulation managed elsewhere (ie, by their cardiologist), did not come to the office for anticoagulation monitoring because of noncompliance, or were otherwise omitted from the anticoagulation log. In addition, this was not a controlled clinical trial with scheduled follow-up and management, and many patients had gaps in their care. Finally, data on warfarin adjustments were not collected before the use of the standardized protocol, so specific comparisons are not possible.

There are several models for management of anticoagulation, including anticoagulation clinics, POC testing, and computer software programs to aid in dose adjustments. Because many family physicians manage anticoagulation in their practice, residents must learn how to effectively care for these patients. Referral of patients receiving warfarin to a pharmacist- or nursemanaged anticoagulation clinic from a residency practice would prevent physicians in training from acquiring this medical knowledge. This quality improvement initiative therefore focused on use of POC testing and a standardized protocol in an effort to simulate the structured approach to managing warfarin therapy provided in an anticoagulation clinic. Our adoption of this intervention resulted in a significant improvement in anticoagulation management, particularly with the implementation of the standardized protocol. It was not enough to switch to POC monitoring. Further adoption of the protocol and 
a more appropriate frequency of follow-up care may lead to an even greater improvement in the management of INR values. Overall, this model represents an intervention in anticoagulation management that could be easily implemented in any family medicine office.

To read or post commentaries in response to this article, see it online at http://www.annfammed.org/cgi/content/full/6/suppl_1/s28.

Key words: Anticoagulation; warfarin; dosing; international normalized ratio; venous thromboembolism; point-of-care systems; bedside testing; protocol; primary care

Submitted March 6, 2007; submitted, revised, July 11, 2007; accepted August 2, 2007.

Prior presentation: Carek PJ, Dickerson LM, Steyer T, Diaz V, Franke C. Scholarly activity for family medicine residents: a history of success. Seminar at the Society of Teachers of Family Medicine Annual Meeting, San Francisco, California; April 2006.

\section{References}

1. White RH. The epidemiology of venous thromboembolism. Circulation. 2003;107(23 Suppl 1):1-4 to 1-8.

2. Singer DE, Albers GW, Dalen JE, Go AS, Halperin JL, Manning WJ. Antithrombotic therapy in atrial fibrillation: the Seventh ACCP Conference on Antithrombotic and Thrombolytic Therapy. Chest. 2004;126(3 Suppl):429S-456S.

3. Ansell J, Hirsh J, Poller L, Bussey H, Jacobson A, Hylek E. The pharmacology and management of the vitamin $\mathrm{K}$ antagonists: the Seventh ACCP Conference on Antithrombotic and Thrombolytic Therapy. Chest. 2004;126(3 Suppl):204S-233S.

4. Salem DN, Stein PD, Al-Ahmad A, et al. Antithrombotic therapy in valvular heart disease-native and prosthetic: the Seventh ACCP Conference on Antithrombotic and Thrombolytic Therapy. Chest. 2004;126(3 Suppl):457S-482S.

5. Samsa GP, Matchar DB, Goldstein LB, et al. Quality of anticoagulation management among patients with atrial fibrillation: results of a review of medical records from 2 communities. Arch Intern Med. 2000;160(7):967-973.

6. Kearon C, Ginsberg JS, Kovacs MJ, et al. Comparison of low-intensity warfarin therapy with conventional-intensity warfarin therapy for long-term prevention of recurrent venous thromboembolism. N Engl J Med. 2003;349(7):631-639.

7. Kearon C, Gent M, Hirsh J, et al. A comparison of three months of anticoagulation with extended anticoagulation for a first episode of idiopathic venous thromboembolism. N Engl J Med. 1999;340(12):901-907.

8. Agnelli G, Prandoni P, Santamaria MG, et al. Three months versus one year of oral anticoagulant therapy for idiopathic deep venous thrombosis. Warfarin Optimal Duration Italian Trial Investigators. N Engl J Med. 2001;345(3):165-169.

9. Bleeding during antithrombotic therapy in patients with atrial fibrillation. The Stroke Prevention in Atrial Fibrillation Investigators. Arch Intern Med. 1996;156(4):409-416.

10. Shermock KM, Bragg L, Connor JT, Fink J, Mazzoli G, Kottke-Marchant K. Differences in warfarin dosing decisions based on international normalized ratio measurements with two point-of-care testing devices and a reference laboratory measurement. Pharmacotherapy. 2002;22(11):1397-1404.
11. Wurster M, Doran T. Anticoagulation management: a new approach. Dis Manag. 2006;9(4):201-209.

12. Poller L, Shiach CR, MacCallum PK, et al. Multicentre randomised study of computerised anticoagulant dosage. European Concerted Action on Anticoagulation. Lancet. 1998;352(9139):1505-1509.

13. Cromheecke ME, Levi M, Colly LP, et al. Oral anticoagulation selfmanagement and management by a specialist anticoagulation clinic: a randomised cross-over comparison. Lancet. 2000;356(9224): 97-102.

14. Cortelazzo S, Finazzi G, Viero P, et al. Thrombotic and hemorrhagic complications in patients with mechanical heart valve prosthesis attending an anticoagulation clinic. Thromb Haemost. 1993;69(4):316-320.

15. Chiquette E, Amato MG, Bussey HI. Comparison of an anticoagulation clinic with usual medical care: anticoagulation control, patient outcomes, and health care costs. Arch Intern Med. 1998;158(15):1641-1647.

16. Ebell MH. Evidence-based initiation of warfarin (Coumadin). Am Fam Physician. 2005;71(4):763-765.

17. Wilson SJ, Wells PS, Kovacs MJ, et al. Comparing the quality of oral anticoagulant management by anticoagulation clinics and by family physicians: a randomized controlled trial. CMAJ. 2003; 169(4):293-298.

18. Gill JM, Landis MK. Benefits of a mobile, point-of-care anticoagulation therapy management program. Jt Comm J Qual Improv. 2002;28(11):625-630.

19. Fitzmaurice DA, Murray ET, Gee KM, Allan TF, Hobbs FD. A randomised controlled trial of patient self management of oral anticoagulation treatment compared with primary care management. J Clin Pathol. 2002;55(11):845-849.

20. Gage BF. Pharmacogenetics-based coumarin therapy. Hematol Am Soc Hematol Educ Program. 2006(1):467-473.

21. Sunderji R, Gin K, Shalansky K, et al. Clinical impact of point-ofcare vs laboratory measurement of anticoagulation. Am J Clin Pathol. 2005;123(2):184-188.

22. McBane RD 2nd, Felty CL, Hartgers ML, Chaudhry R, Beyer LK Santrach PJ. Importance of device evaluation for point-of-care prothrombin time international normalized ratio testing programs. Mayo Clin Proc. 2005;80(2):181-186.

23. Reiss RA, Haas CE, Griffis DL, Porter B, Tara MA. Point-of-care versus laboratory monitoring of patients receiving different anticoagulant therapies. Pharmacotherapy. 2002;22(6):677-685.

24. van den Besselaar AM. Accuracy, precision, and quality control for point-of-care testing of oral anticoagulation. J Thromb Thrombolysis. 2001;12(1):35-40.

25. Jackson SL, Peterson GM, House M, Bartlett T. Point-of-care monitoring of anticoagulant therapy by rural community pharmacists: description of successful outcomes. Aust J Rural Health. 2004;12(5):197-200.

26. Jackson SL, Peterson GM, Vial JH, Jupe DM. Improving the outcomes of anticoagulation: an evaluation of home follow-up of warfarin initiation. J Intern Med. 2004;256(2):137-144.

27. Woods K, Douketis JD, Schnurr T, Kinnon K, Powers P, Crowther MA. Patient preferences for capillary vs. venous INR determination in an anticoagulation clinic: a randomized controlled trial. Thromb Res. 2004;114(3):161-165.

28. MacMillan D. Reimbursement for point of care testing. Point of Care. 2002;1(4):253-258.

29. Dalere GM, Coleman RW, Lum BL. A graphic nomogram for warfarin dosage adjustment. Pharmacotherapy. 1999;19(4):461-467. 